\title{
Fidelity approach to Gaussian transitions
}

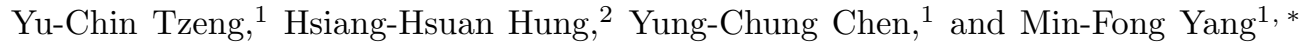 \\ ${ }^{1}$ Department of Physics, Tunghai University, Taichung, Taiwan \\ ${ }^{2}$ Department of Physics and Astronomy, University of Pittsburgh, Pittsburgh, Pennsylvania 15260
}

(Dated: October 31, 2018)

\begin{abstract}
The fidelity approach to the Gaussian transitions in spin-one $X X Z$ spin chains with three different values of Ising-like anisotropy $\lambda$ is analyzed by means of the density matrix renormalization group (DMRG) technique for systems of large sizes. We find that, despite the success in the cases of $\lambda=2.59$ and 1 , the fidelity susceptibility fails to detect the Gaussian transition for $\lambda=0.5$. Thus our results demonstrate the limitation of the fidelity susceptibility in characterizing quantum phase transitions, which was proposed recently in general frameworks.
\end{abstract}

PACS numbers: 03.67.-a, 75.10.Pq, 05.70.Fh, 71.10.Pm,

\section{INTRODUCTION}

Due to latest progress in quantum information science [1, people attempt to investigate the quantum phase transitions (QPTs) 2] in many-body systems from the perspective of quantum information [3]. Recently, it was suggested that the ground-state fidelity, a basic notion that emerged from quantum information science, should be able to signal the QPTs [4, 5]. Because the fidelity is a measure of distinguishability between two states, one anticipates that the ground-state fidelity typically drops abruptly at the quantum critical point, as a consequence of the dramatic changes in the structure of the ground states when systems undergo a QPT. Thus the fidelity should be able to furnish a signature of the quantum critical point. Another related transition indicator is provided by the singularity in the second derivative of the fidelity [6, 7, [8] (or the so-called "fidelity susceptibility" [6]). The fidelity approach has been tested for various many-body systems $4,5,6,7,8,9,10,11,12,13,14,15,16,17$, $18,19,20,21,22,23,24,25,26,27,28,29,30,31,32$. Since the fidelity is a purely Hilbert-space geometrical quantity, no a priori knowledge of the structure (order parameter, correlations driving the QPTs, topology, etc.) of the considered system is required for its use. Therefore, the fidelity analysis might be a potential universal tool for characterizing the QPTs. That is the reason why the investigation of the role of fidelity in detecting the topological phase transition, where no local order parameter can distinguish the ground states around the critical points, has started quite recently [29, 30, 31, 32].

Besides the examination of its validity in restricted situations, general understanding of the fidelity approach has come out, and the limitation of the fidelity susceptibility in detecting QPTs has been established [19, 21]. Conventionally, QPTs are characterized by the nonanalyticities of the ground-state energy: first-order QPTs

*Electronic address: mfyang@thu.edu.tw
(1QPTs) are characterized by discontinuity in the first derivative of the ground-state energy, second-order QPTs (2QPTs) are characterized by discontinuity/singularity in the second derivative, and so on. It is pointed out that, while the fidelity susceptibility is indeed an effective tool in detecting the critical points of 1QPTs and 2QPTs, it may fail to detect higher-order QPTs 21]. Moreover, the authors in Ref. [19] show that, for critical (gapless) systems of finite size $L$, the fidelity susceptibility $\mathcal{S}$ fulfills scaling relations

$$
\mathcal{S} \sim L^{-\Delta_{Q}}, \quad \Delta_{Q}=2 \Delta_{V}-2 z-d,
$$

where $d$ is the spatial dimension, $z$ is the dynamic exponent, and $\Delta_{V}$ is the scaling dimension of the transitiondriving term in the Hamiltonian. The scaling relation in Eq. (11) implies that, for critical points with $\Delta_{Q}>0, \mathcal{S}$ is non-singular even in the thermodynamic limit. Therefore, the fidelity susceptibility is not suitable to single out these QPTs.

Due to the lack of knowledge of exact ground state wave functions, the ground-state fidelity is usually difficult to be calculated. For models that are not exactly solvable, most of researchers resort to numerical exact diagonalization on small systems. However, because of finite-size effects, the relevance of their results for the thermodynamic limit may not be guaranteed. Therefore, further investigations for systems of large sizes are clearly called for. By using the density matrix renormalization group (DMRG) technique 33], the fidelity susceptibility of the spin-one $X X Z$ spin chains with an on-site anisotropic term for the case of the Ising-like anisotropy parameter $\lambda=1$ is evaluated for system sizes up to $L=160$ [23]. The scaling relation in Eq. (11) has been confirmed numerically for both the Ising and the Gaussian transitions of this model.

In the present article, we further pursue the fidelity approach by analyzing the Gaussian transitions of the same model for various parameters of Ising-like anisotropy up to $L=400$. Because the critical exponents of the Gaussian transitions change continuously along the critical lines, the QPTs of different order can be realized simply by choosing different Ising-like anisotropy parameter 
$\lambda$. Here, we consider three cases of $\lambda=2.59,1$, and 0.5 , which correspond to 2QPT, third-order QPT (3QPT), and fifth-order QPT (5QPT), respectively (see Secs. II and (III). We find that, while the fidelity susceptibility can serve as a valid transition indicator for the Gaussian transitions in the cases of $\lambda=2.59$ and 1 , it does not show any singularity around the critical point for $\lambda=0.5$ and fails to detect this higher-order QPT. Therefore, our results provide a concrete illustration for the failure of the fidelity susceptibility in characterizing QPTs, and lend further numerical supports of the general proposals in Refs. [19] and [21].

This paper is organized as follows. The model Hamiltonian and its low-energy effective theory, as well as the general scaling arguments, are described in Sec. II] Our DMRG calculations for the fidelity susceptibility are presented in Sec. III We summarize and conclude our results in Sec. IV] The appendix contains an outline of the computation of the ground-state fidelity under DMRG algorithm.

\section{SPIN-ONE ANISOTROPIC MODEL AND ITS EFFECTIVE THEORY}

The $S=1 X X Z$ chains with uniaxial single-ion-type anisotropy is defined by the Hamiltonian:

$$
H=\sum_{j}\left\{S_{j}^{x} S_{j+1}^{x}+S_{j}^{y} S_{j+1}^{y}+\lambda S_{j}^{z} S_{j+1}^{z}+D\left(S_{j}^{z}\right)^{2}\right\},
$$

where $S_{j}^{\alpha}(\alpha=x, y, z)$ stand for the spin-one operators at the $j$-th lattice site. $\lambda$ and $D$ parametrize the Ising-like and the uniaxial single-ion anisotropies, respectively. The full phase diagram consists of six different phases [34, 35] (see Refs. [36, 37, 38, 39] for recent numerical determinations). Here we focus our attention on the Gaussian transitions between the Haldane and the large- $D$ phases. It has been found that the low-energy effective continuum theory for the Gaussian transitions can be described by the sine-Gordon model [37, 38] (here we follow the notations used in Ref. [38])

$$
H_{S G}=\frac{1}{2}\left[\Pi^{2}+\left(\partial_{x} \Phi\right)^{2}\right]-\frac{\mu}{a^{2}} \cos (\sqrt{4 \pi K} \Phi)
$$

where $\Pi$ and $\Phi$ are the conjugate bosonic phase fields, and $a$ is a short-distance cut-off of the order of the lattice spacing. The coefficient $\mu \propto\left(D-D_{c}\right)$ in the vicinity of the critical point $D_{c}$ for a given $\lambda$, and thus becomes zero at the transition point. The value of the Luttinger liquid parameter $K$ varies continuously between $1 / 2$ and 2 along the critical line. Because all the scaling dimensions and the critical exponents are determined by a single parameter $K$, they also change continuously along the critical line. From the sine-Gordon theory [40], it is found that the critical exponent of the correlation length

$$
\nu=\frac{1}{2-K}
$$

and the scaling dimension $\Delta_{V}=K$ for the transitiondriving term $\cos (\sqrt{4 \pi K} \Phi)$. Thus the exponent in the scaling relation of the fidelity susceptibility in Eq. (1) becomes ( $d=1$ and $z=1$ are assumed here)

$$
\Delta_{Q}=2 K-3
$$

From this expression, one realizes immediately that, for critical points with $K>3 / 2, \Delta_{Q}$ is positive and then $\mathcal{S}$ becomes non-singular even in the thermodynamic limit. That is, the fidelity susceptibility fails to single out the Gaussian transitions with $K>3 / 2$.

According to the conventional classification, a 1QPT is characterized by a finite discontinuity in the first derivative of the ground state energy. Similarly, a 2QPT is characterized by a finite discontinuity, or divergence, in the second derivative of the ground state energy, assuming the first derivative is continuous. From the effective theory in Eq. (3) and the scaling hypothesis, the first derivative of the ground-state energy density $e(D)$ with respect to $D$ at a fixed $\lambda$ gives [38]

$$
\begin{aligned}
\frac{\partial e(D)}{\partial D} & \sim\langle\cos (\sqrt{4 \pi K} \Phi)\rangle \\
& \sim \operatorname{sgn}\left(D-D_{c}\right)\left|D-D_{c}\right|^{\rho}
\end{aligned}
$$

where

$$
\rho=\nu \Delta_{V}=\frac{K}{2-K}
$$

From Eq. (6), the second derivative of $e(D)$ can be obtained as

$$
\frac{\partial^{2} e(D)}{\partial D^{2}} \sim\left|D-D_{c}\right|^{\rho-1}
$$

Therefore, the second derivative of $e(D)$ shows a divergence for $0<\rho<1$ (or $0<K<1$ ). In this case, the Gaussian transition corresponds to a 2QPT. Similarly, for $1<\rho<2$ (or $1<K<4 / 3$ ), the Gaussian transition can be classified as a $3 \mathrm{QPT}$. Finally, as mentioned above, for the Gaussian transitions with $K>3 / 2$, the fidelity susceptibility fails to detect them. In these cases, their values of $\rho$ will be greater than three, and these transitions will be identified as the QPTs of higher than forth order.

\section{DMRG RESULTS}

In the present work, we consider three cases of $\lambda=$ 2.59, 1 , and 0.5 , whose Luttinger liquid parameter has been found to be $K=0.85,1.328$, and 1.580 , respectively [37, 39]. From the discussions in the previous section, the QPTs of these three cases should correspond to 2QPT, 3QPT, and 5QPT, respectively. Therefore, one expects that the fidelity susceptibility will not show singularity for the case of $\lambda=0.5$ only. 
In the following, our DMRG results are presented in order. The fidelity susceptibility for system in Eq. (2) of size $L$ is calculated by 10,15 ]

$$
\mathcal{S}(D)=\lim _{\delta \rightarrow 0} \frac{2[1-\mathcal{F}(D, D+\delta)]}{L \delta^{2}},
$$

where the ground-state fidelity is given by [5]

$$
\mathcal{F}(D, D+\delta)=\left|\left\langle\Psi_{0}(D) \mid \Psi_{0}(D+\delta)\right\rangle\right|
$$

with $\left|\Psi_{0}(D)\right\rangle$ and $\left|\Psi_{0}(D+\delta)\right\rangle$ being two normalized ground states corresponding to neighboring Hamiltonian parameters. Details on the computation of the groundstate fidelity under DMRG algorithm is explained in the appendix. In our calculations, $\delta=10^{-3}$ is used. Our results are evaluated by means of the finite-system DMRG technique under open boundary conditions for system sizes up to $L=400$, where 300 states per block are kept and five DMRG sweeps are performed for the truncation error being $10^{-9}$ at most. Finally, in order to select a specific ground state and stabilize our calculations of the fidelity around the Néel phase, a Zeeman term $h_{1} S_{1}^{z}$ acting only upon the spin at site 1 is added in the Hamiltonian of Eq. (2). Here we take the boundary magnetic field $h_{1}=-1$.

\section{A. case of $\lambda=2.59$}

The findings of the fidelity susceptibility $\mathcal{S}(D)$ and the ground-state fidelity $\mathcal{F}(D, D+\delta)$ for $\lambda=2.59$ are shown in Fig. 1(a). As shown in the inset, drops in the groundstate fidelity are observed, which signal precursors of the Gaussian transition in the model under consideration. Further evidences for indicating QPTs are provided by the results of the fidelity susceptibility $\mathcal{S}$ and the second derivative of the ground-state energy density $\partial^{2} e / \partial D^{2}$. As seen from Fig. 11, the maximum values of both $\mathcal{S}$ and $-\partial^{2} e / \partial D^{2}$ grow with increasing size, and thus indicate divergence in the $L \rightarrow \infty$ limit (see also Fig. 3 below). As discussed in Sec. III, the divergent behaviors both in the fidelity susceptibility and the second derivative of the ground-state energy density indicate that the transition we found should be a $2 \mathrm{QPT}$.

According to the finite-size scaling theory [41], one has

$$
\left|D_{\max }(L)-D_{c}\right| \propto L^{-1 / \nu}
$$

where $D_{c}$ is the critical point in the thermodynamic limit and $\nu$ is the critical exponent of the correlation length. Thus $D_{c}$ can be determined by an extrapolation procedure from the locations $D_{\max }(L)$ of the local maxima in $\mathcal{S}$ and $-\partial^{2} e / \partial D^{2}$ on a size- $L$ system. The results for $\lambda=2.59$ is shown in Fig. 2. We find that $D_{c} \simeq 2.293$ and $\nu \simeq 0.79$ for the findings of $\mathcal{S}$, while $D_{c} \simeq 2.294$ and $\nu \simeq 0.77$ for the data of $-\partial^{2} e / \partial D^{2}$. Because of the relation in Eq. (4), the Luttinger liquid parameter $K \simeq 0.74$ $(K \simeq 0.70)$ for the data related to $\mathcal{S}\left(-\partial^{2} e / \partial D^{2}\right)$. We

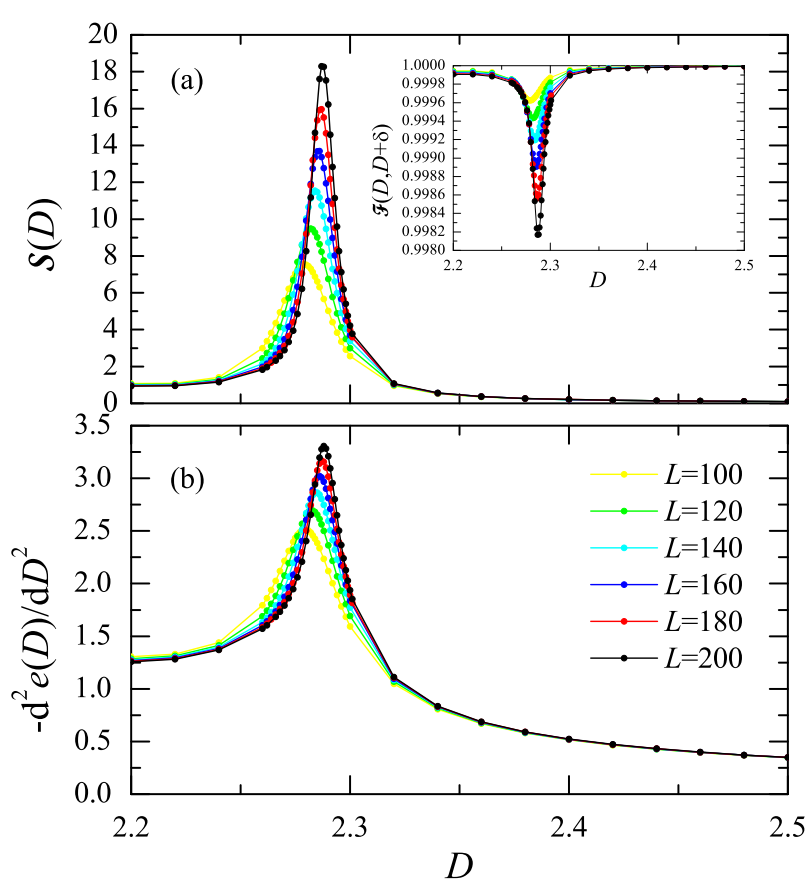

FIG. 1: (Color online) (a) Fidelity susceptibility $\mathcal{S}$, (b) the second derivative of the ground-state energy density $-\partial^{2} e / \partial D^{2}$ as functions of $D$ for various sizes $L$ with $\lambda=2.59$. Inset in the top panel shows the fidelity $\mathcal{F}(D, D+\delta)$ as functions of $D$ for the corresponding sizes. Here we take $\delta=10^{-3}$.

find that our results are consistent with the previous findings [37], where $D_{c} \simeq 2.30$ and $K \simeq 0.85$. We note that the DMRG calculations in Refs. 37] and [39] are under the periodic boundary conditions, rather than the open boundary conditions used in the present work. The small discrepancy of our results from theirs may be due to the different boundary conditions employed.

Moreover, at the critical point, where the only length scale is provided by the system size itself, the scaling formula in Eq. (8) implies

$$
\frac{\partial^{2} e(D)}{\partial D^{2}} \sim L^{-(\rho-1) / \nu} .
$$

From Eqs. (4) and (7), one has

$$
-(\rho-1) / \nu=2(1-K) \text {. }
$$

On the other hand, the fidelity susceptibility $\mathcal{S}$ is proposed to fulfill the scaling relation in Eq. (1). To verify the predicted critical scaling behaviors in Eqs. (1) and (12), the values $\mathcal{S}_{\max }$ and $\left[-\partial^{2} e / \partial D^{2}\right]_{\max }$ of the local maxima in $\mathcal{S}$ and $-\partial^{2} e / \partial D^{2}$ for various sizes $L$ are plotted in Figs. 3(a) and (b), respectively. It is found that our data do fulfill the predicted critical scaling behaviors with $\Delta_{Q} \simeq-1.28$ and $-(\rho-1) / \nu \simeq 0.40$. From Eqs. (5) and (13), our values of $\Delta_{Q}$ and $-(\rho-1) / \nu$ gives $K \simeq 0.86$ and 0.80 , respectively. The Luttinger liquid parameter $K$ determined by the present finite-size scaling agrees with the 


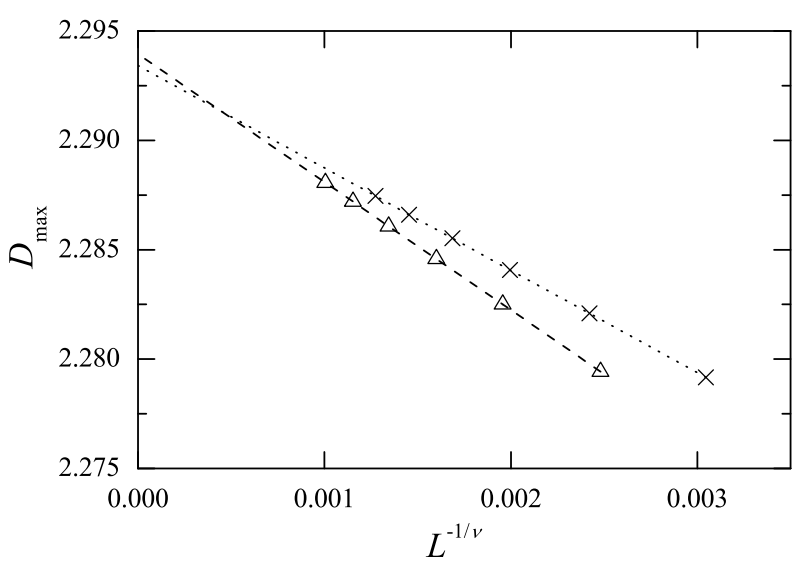

FIG. 2: Finite-size scaling of $D_{\max }$ of $\mathcal{S}(\times)$ and $-\partial^{2} e / \partial D^{2}$ $(\triangle)$ versus $L^{-1 / \nu}$ for $\lambda=2.59$. The lines are least square fits, where $\nu \simeq 0.79(\nu \simeq 0.77)$ for those $D_{\text {max }}$ 's corresponding to the local maxima in the curves of $\mathcal{S}\left(-\partial^{2} e / \partial D^{2}\right)$.

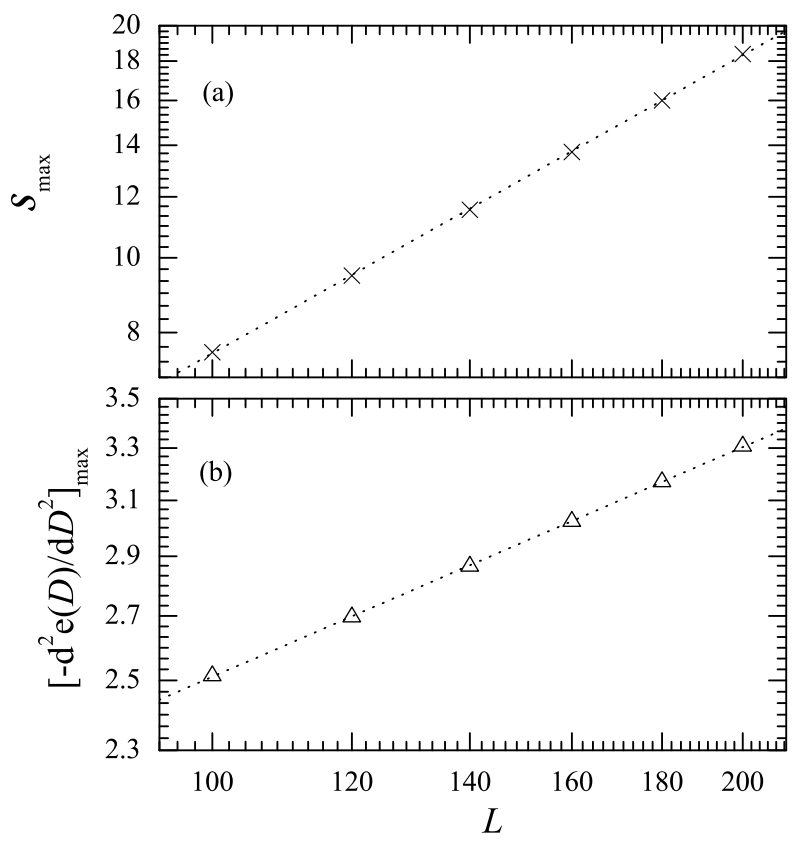

FIG. 3: The log-log plot of (a) $\mathcal{S}_{\max }$ and (b) $\left[-\partial^{2} e / \partial D^{2}\right]_{\max }$ for various sizes $L$ with $\lambda=2.59$. The lines are least square straight line fits.

previous findings [37] and those determined by the critical exponent $\nu$ coming from the scaling in Fig. 2, Thus the fact that a single parameter $K$ controls all the critical exponents for the Gaussian transition is confirmed by our numerical results.

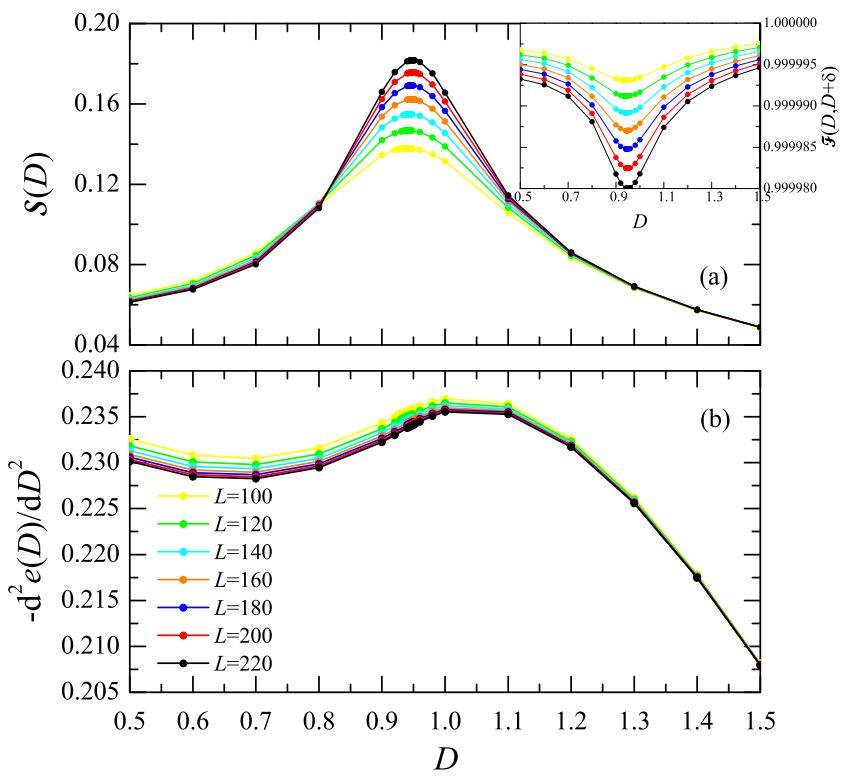

FIG. 4: (Color online) (a) Fidelity susceptibility $\mathcal{S}$, (b) the second derivative of the ground-state energy density $-\partial^{2} e / \partial D^{2}$ as functions of $D$ for various sizes $L$ with $\lambda=1$. Inset in the top panel shows the fidelity $\mathcal{F}(D, D+\delta)$ as functions of $D$ for the corresponding sizes. Here we take $\delta=10^{-3}$.

\section{B. case of $\lambda=1$}

For the case of $\lambda=1$, a numerical support of the use of the fidelity in detecting QPTs has been provided in Ref. [23], and the scaling relation of the fidelity susceptibility in Eq. (11) has also been verified there. Here, we focus our attention on the comparison between the scaling behaviors of the fidelity susceptibility and the second derivative of the ground-state energy density, which is shown in Fig. 4. As observed in Ref. 23], our present data in Fig. 4(a) do show the developing drops in the ground-state fidelity and the divergent behaviors in $\mathcal{S}$. Thus the fidelity susceptibility does signal precursors of the Gaussian transition in the model under consideration. However, as seen from Fig. 4(b), the second derivative of the ground-state energy density does not grow with increasing size in the same parameter regime. It implies that this QPT should be the one of higher than second order. To the author's knowledge, the present model at $\lambda=1$ may be the first concrete example in the literature with a QPT of higher than second order, which can be singled out unambiguously by using the fidelity susceptibility.

\section{C. case of $\lambda=0.5$}

Although the fidelity susceptibility is possible to detect the QPTs of higher than second order, as explained 


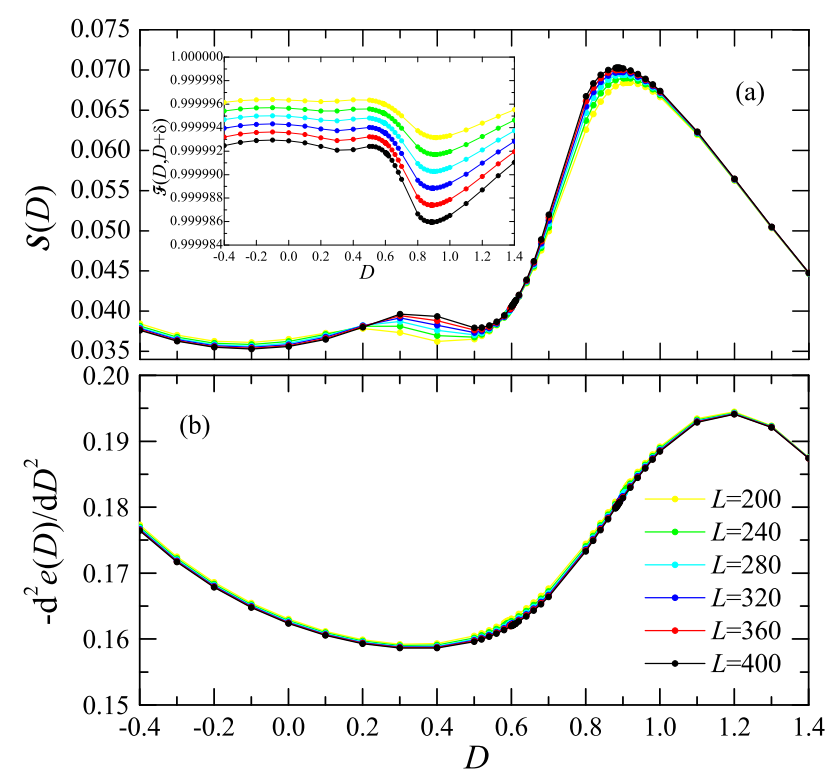

FIG. 5: (Color online) (a) Fidelity susceptibility $\mathcal{S}$, (b) the second derivative of the ground-state energy density $-\partial^{2} e / \partial D^{2}$ as functions of $D$ for various sizes $L$ with $\lambda=0.5$. Inset in the top panel shows the fidelity $\mathcal{F}(D, D+\delta)$ as functions of $D$ for the corresponding sizes. Here we take $\delta=10^{-3}$.

in Ref. 21], it may not always work. For example, as discussed at the end of Sec. III the fidelity susceptibility will fail to detect those Gaussian transitions with the Luttinger liquid parameter $K>3 / 2$, which give $\Delta_{Q}>0$ and correspond to the QPTs of higher than forth order. In the following, we show that the present model at $\lambda=0.5$ provides an example for this case.

Our DMRG results of the fidelity susceptibility $\mathcal{S}(D)$, the ground-state fidelity $\mathcal{F}(D, D+\delta)$, and the second derivative of the ground-state energy density $\partial^{2} e / \partial D^{2}$ for $\lambda=0.5$ are shown in Fig. 5 . While developing drops in the ground-state fidelity are indeed observed in the inset of Fig. 馬(a), the fidelity susceptibility and the second derivative of the ground-state energy density show no divergent behavior up to $L=400$. That is, this Gaussian transition must be a QPT of higher than second order, which the fidelity susceptibility fails to detect. Further support for such a conclusion is provided by the findings of $\mathcal{S}_{\max }$ of the local maxima in $\mathcal{S}$ for various sizes $L$, which is plotted in Fig. 6. It is found that maximal values of $\mathcal{S}$ do tend to saturate, rather than diverge, in the $L \rightarrow \infty$ limit. Thus this Gaussian transition does provide a counterexample of the usefulness of the fidelity susceptibility as an effective tool in detecting QPTs.

One may wonder if the non-singular behavior in the fidelity susceptibility shown in Figs. 5 and 6 is due to the possible finite-size effects, even though sizes up to $L=$ 400 have been considered. By contrast, the entanglement entropy is evaluated below. It has been found that the divergent character of the entanglement entropy can also

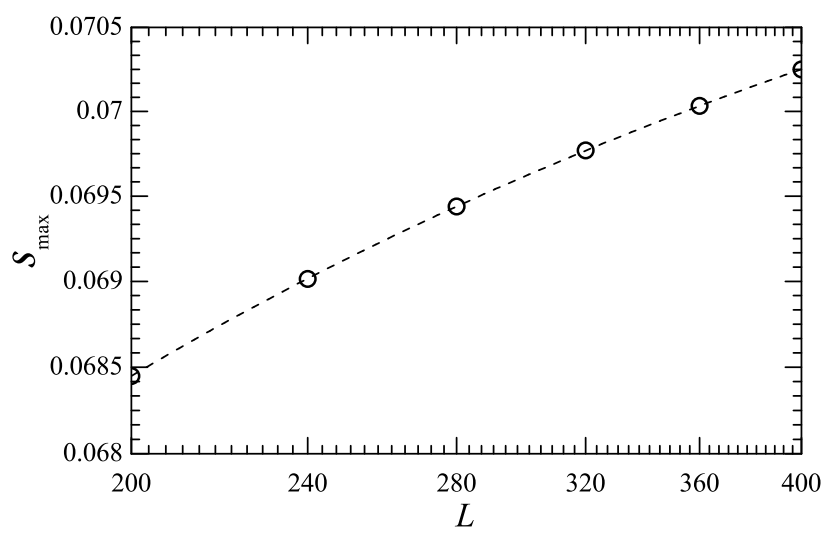

FIG. 6: The log-log plot of $\mathcal{S}_{\max }$ for various sizes $L$ with $\lambda=0.5$. The dashed curve is the least square fit by using the formula $\mathcal{S}_{\max }=\mathcal{S}_{\infty}-a L^{-b}$ with $\mathcal{S}_{\infty}=0.073, a=0.23$, and $b=0.75$.

show the existence of the QPTs [42, 43, 44, 45, 46, 47, 48]. Within the present DMRG calculations, the singular behavior of the entanglement entropy is indeed observed in the given parameter regime (see Fig. 7 below). Thus the desired Gaussian transition does appear there. This indicates that the system sizes we employed should be large enough and finite-size effects should play no important role in our calculations.

Here we consider the entanglement entropy, or the von Neumann entropy of the reduced density matrix $\rho_{R}(D)$ of the right-hand block of $L / 2$ contiguous spins

$$
\mathcal{E}(D)=-\operatorname{Tr}\left[\rho_{R}(D) \log _{2} \rho_{R}(D)\right] .
$$

Our DMRG results are presented in Fig. 7. It is found from Fig. Z(a) that peaks do grow as size $L$ increases, which show the existence of the expected Gaussian transition. Further evidence is provided by the findings of $\mathcal{E}_{\max }$ of the local maxima in $\mathcal{E}$ for various sizes $L$, which is plotted in Fig. 7 (b). It indicates that $\mathcal{E}_{\max } \propto \log _{2}(L)$ and thus $\mathcal{E}_{\max }$ will diverge in the $L \rightarrow \infty$ limit. It is known that the slope in Fig. 7(b) gives the value of $c / 6$ under open boundary conditions [42, 43, 44, 45, 46, 47, 48], where $c$ is the central charge of the conformal field theory. Our data give $c \simeq 0.98$, which agrees with the theoretical value $c=1$ for the Gaussian transition [37]. Furthermore, according to Eq. (11), the corresponding critical points $D_{c}$ can be deduced from the locations $D_{\max }(L)$ of the local maxima in $\mathcal{E}(D)$ on a size- $L$ system, as shown in Fig. 7(c). It is found that $D_{c} \simeq 0.63$ and $\nu \simeq 1.51$. The value of $\nu$ implies the Luttinger liquid parameter $K \simeq 1.34$ due to the relation in Eq. (44). In Ref. [37], $D_{c} \simeq 0.65$ and $K \simeq 1.580$ are reported. Thus our results are again consistent with their findings. As mentioned before, the discrepancy of our results from theirs may be due to the different boundary conditions employed. In short, the missing QPT in the measurements of the fidelity susceptibility is able to be captured by using the 

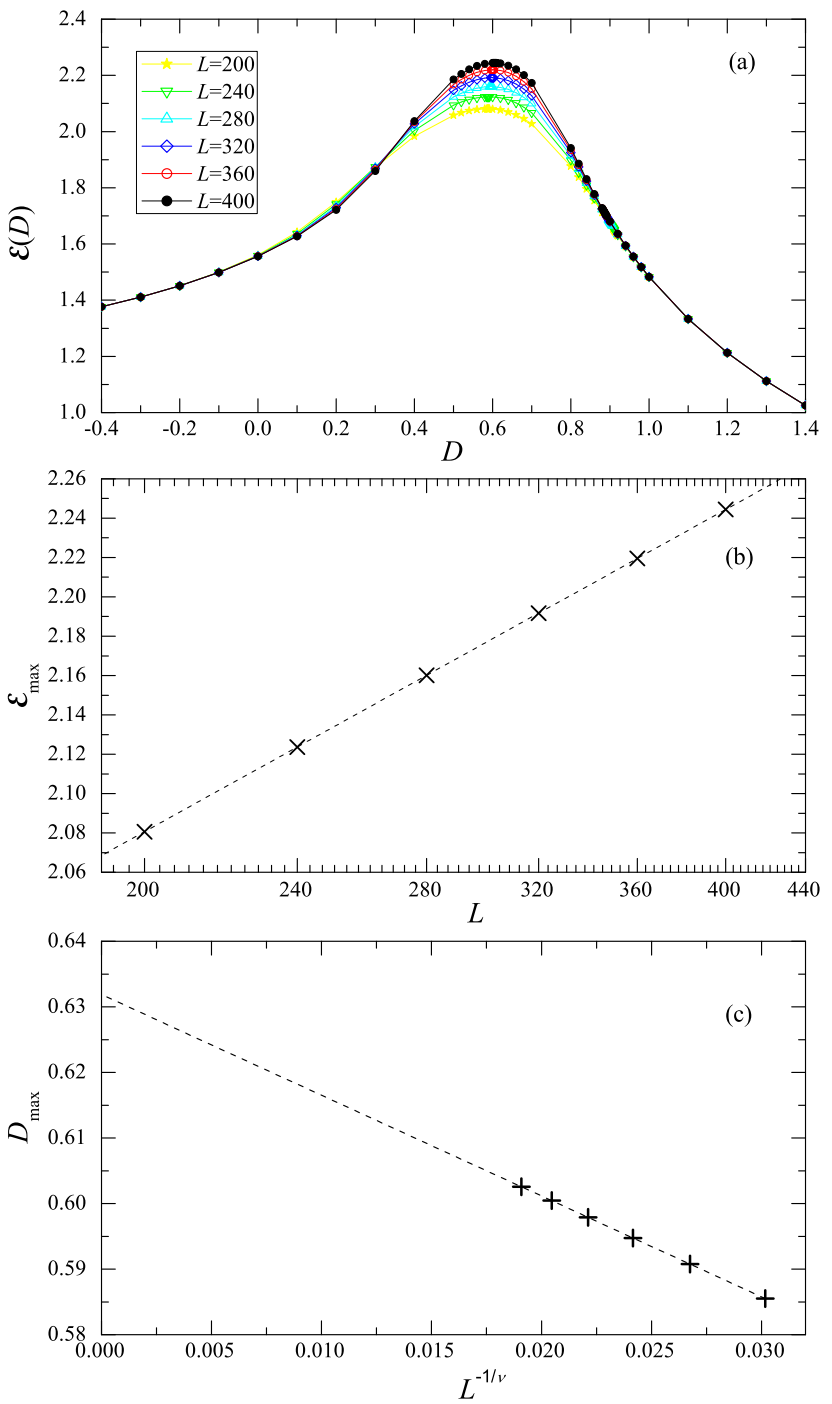

FIG. 7: (Color online) (a) Entanglement entropy $\mathcal{E}$ as functions of $D$ for various sizes $L$. (b) The semi-log plot of $\mathcal{E}_{\text {max }}$ for various sizes $L$. The line is least square straight line fit. (c) Finite-size scaling of $D_{\max }$ of $\mathcal{E}$ versus $L^{-1 / \nu}$. The line is least square fit with $\nu \simeq 1.51$. Here $\lambda=0.5$.

tool of the entanglement entropy.

\section{CONCLUSIONS}

In summary, we present numerical analysis of the fidelity approach to the Gaussian transitions in the spinone $X X Z$ spin chains in Eq. (2) with three different values of Ising-like anisotropy $\lambda$. It is found that the fidelity susceptibility can detect the Gaussian transitions for the cases of $\lambda=2.59$ and 1 . As in the case of $\lambda=1$ investigated in Ref. [23], the scaling relation of the fidelity susceptibility proposed in Ref. [19] is again verified by the present DMRG calculations for the case of $\lambda=2.59$.
Moreover, the critical point and some of the corresponding critical exponents are determined through a proper finite-size scaling analysis, and these values agree with the findings reported in the literature. Although it is successful in characterizing the QPTs for the cases of $\lambda=2.59$ and 1 , the fidelity susceptibility is not able to detect the Gaussian transitions for $\lambda=0.5$. This observation can be explained by the general theories in Refs. 19] and [21]. Thus our work provides an instance for the limitation of the use of the fidelity susceptibility for revealing QPTs.

Finally, we remind that developing drops in the ground-state fidelity are observed around the critical point even for the case of $\lambda=0.5$ [see the inset of Fig. [5(a)]. This seems to indicate that the singularity of the fidelity may still be a valid transition indicator even for higher-order QPTs. The failure of its second derivatives (say, the fidelity susceptibility) may just imply that higher derivatives of the fidelity are necessary to signal some higher-order QPTs. Indeed, such a conclusion can be deduced from the general arguments in Refs. [19] and 21]. It was shown in Ref. [21] that the fidelity susceptibility $\mathcal{S}$ (or the second derivative of the fidelity per site) can be associated with the first derivative of the density matrix $\rho_{0}$ of the ground state [see Eq. (6) therein]. Thus $\mathcal{S}$ can not detect the higher-order QPTs resulting from the nonanalyticities in second (and even higher-order) derivatives of $\rho_{0}$. That is, these higherorder QPTs can be recognized only by the singular behavior in higher-order derivatives of the fidelity. Besides, the singular part of $\mathcal{S}$ has been shown in Ref. [19] to behave as $\mathcal{S} \sim\left|D-D_{c}\right|^{\nu \Delta_{Q}}$. Therefore, the third derivative of the fidelity should behave as $\left|D-D_{c}\right|^{\nu \Delta_{Q}-1}$. This implies that, although the second derivative of the fidelity (or the fidelity susceptibility $\mathcal{S}$ ) does not diverge for the Gaussian transitions with $3 / 2<K<5 / 3$ [thus $0<\nu \Delta_{Q}<1$, see Eqs. (4) and (5)], the third derivative does become singular at these critical points. That is, one does need the third derivative of the fidelity to single out these higher-order QPTs. The more complete and deeper understanding of the role played by the fidelity and its derivatives in the studying of the higher-order QPTs is an interesting issue which calls for future investigations.

\section{Acknowledgments}

YCT and YCC are grateful for the support from the National Science Council of Taiwan under NSC 96-2112M-029-003-MY3. MFY acknowledge the support by the National Science Council of Taiwan under NSC 96-2112M-029-004-MY3. 


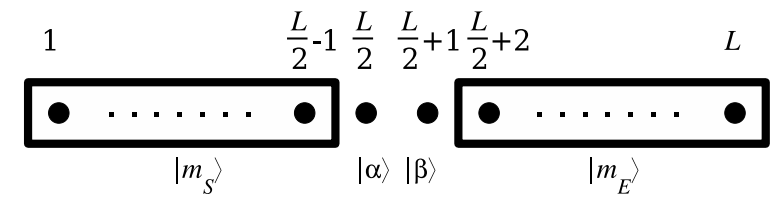

FIG. 8: The configuration of wave functions in the superblock.

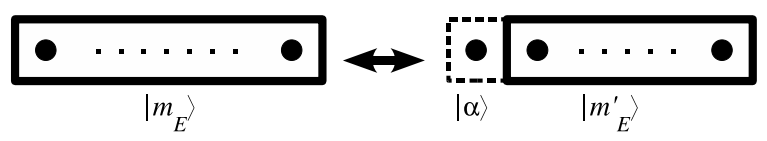

FIG. 9: The basis transformation between a block and an enlarged block.

\section{APPENDIX: COMPUTATION OF FIDELITY UNDER DMRG ALGORITHM}

The DMRG method had been used to calculate the overlap of two different ground states for a decade. For example, people had applied this technique to evaluate the exponent of the orthogonality catastrophe for the problem of a single impurity in a one-dimensional Luttinger liquid [49]. Recently, this method is used to investigate the scaling properties of fidelity susceptibility for the model in Eq. (2) with $\lambda=1$ [23]. For completeness, the algorithm within the DMRG framework of computation of the ground-state fidelity (i.e., the overlap between two ground states corresponding to two different system parameters) is presented in this appendix. Similar discussions in terms of the formalism of matrix product states can be found at the end of Sec. 2.1 in Ref. [50].

Let $|\Phi\rangle$ and $|\Psi\rangle$ be the normalized wave functions obtained from two different DMRG calculations with distinct Hamiltonian parameters for systems of the same size. Assume that the same number of states $m$ is kept in both DMRG calculations. Moreover, both wave functions are expressed in the same scheme, where the corresponding system (and thus the corresponding environment) blocks are of the same length. Therefore, these two wave functions can be written as (see Fig. 8)

$$
\begin{gathered}
|\Psi\rangle=\sum_{m_{S}, \alpha, \beta, m_{E}} \Psi_{m_{S}, \alpha, \beta, m_{E}}\left|m_{S}\right\rangle|\alpha\rangle|\beta\rangle\left|m_{E}\right\rangle, \\
|\Phi\rangle=\sum_{\bar{m}_{S}, \alpha^{\prime}, \beta^{\prime}, \bar{m}_{E}} \Phi_{\bar{m}_{S}, \alpha^{\prime}, \beta^{\prime}, \bar{m}_{E}}\left|\bar{m}_{S}\right\rangle\left|\alpha^{\prime}\right\rangle\left|\beta^{\prime}\right\rangle\left|\bar{m}_{E}\right\rangle .
\end{gathered}
$$

Here $|\alpha\rangle\left(\left|\alpha^{\prime}\right\rangle\right)$ and $|\beta\rangle\left(\left|\beta^{\prime}\right\rangle\right)$ denote the states in the local Hilbert space of dimension $s$ at sites $L / 2$ and $L / 2+1$, respectively. $\left|m_{S}\right\rangle$ and $\left|\bar{m}_{S}\right\rangle$ represent the $m$ renormalized states of the system blocks, while $\left|m_{E}\right\rangle$ and $\left|\bar{m}_{E}\right\rangle$ are those $m$ renormalized states of the environment blocks. Thus the overlap between these two ground states becomes

$$
\langle\Phi \mid \Psi\rangle=\sum_{\bar{m}_{S}, \alpha^{\prime}, \beta^{\prime}, \bar{m}_{E} ; m_{S}, \alpha, \beta, m_{E}} \Phi_{\bar{m}_{S}, \alpha^{\prime}, \beta^{\prime}, \bar{m}_{E}}^{*}\left\langle\bar{m}_{S} \mid m_{S}\right\rangle\left\langle\alpha^{\prime} \mid \alpha\right\rangle\left\langle\beta^{\prime} \mid \beta\right\rangle\left\langle\bar{m}_{E} \mid m_{E}\right\rangle \Psi_{m_{S}, \alpha, \beta, m_{E}}
$$

Remind that $\left\langle\alpha^{\prime} \mid \alpha\right\rangle$ and $\left\langle\beta^{\prime} \mid \beta\right\rangle$ are nothing but the matrix elements of the $s \times s$ identity matrix $\hat{1}$ in the subspaces of the local Hilbert spaces at sites $L / 2$ and $L / 2+1$, respectively. Besides, let's define

$$
\begin{aligned}
{\left[\hat{1}_{\frac{L}{2}-1}^{S}\right]_{\bar{m}_{S}, m_{S}} } & =\left\langle\bar{m}_{S} \mid m_{S}\right\rangle, \\
{\left[\hat{1}_{\frac{L}{2}-1}^{E}\right]_{\bar{m}_{E}, m_{E}} } & =\left\langle\bar{m}_{E} \mid m_{E}\right\rangle,
\end{aligned}
$$

which serve as the matrix elements of the $m \times m$ "identity matrices" $\left[\hat{1}_{\frac{L}{2}-1}^{S}\right]$ and $\left[\hat{1}_{\frac{L}{2}-1}^{E}\right]$ of the system and the environment blocks of length $\frac{L}{2}-1$, respectively. Therefore, the expression of the overlap in Eq. A.3 can be rewritten as the following compact from

$$
\langle\Phi \mid \Psi\rangle=\langle\langle\Phi||[\hat{1}] \| \Psi\rangle\rangle
$$

with

$$
[\hat{1}]=\left[\hat{1}_{\frac{L}{2}-1}^{S}\right] \otimes \hat{1} \otimes \hat{1} \otimes\left[\hat{1}_{\frac{L}{2}-1}^{E}\right],
$$

where ||$\Phi\rangle\rangle$ and $\| \Psi\rangle\rangle$ represent the column vectors of dimension $s^{2} m^{2}$ with the components $\Phi_{\bar{m}_{S}, \alpha^{\prime}, \beta^{\prime}, \bar{m}_{E}}$ and
$\Psi_{m_{S}, \alpha, \beta, m_{E}}$, respectively. Now the remaining work of evaluation of fidelity reduces to find a general strategy of calculating the two $m \times m$ matrices $\left[\hat{1}_{\frac{L}{2}-1}^{S}\right]$ and $\left[\hat{1}_{\frac{L}{2}-1}^{E}\right]$.

Note that the renormalized states of the environment blocks of length $l$ can be expressed as (see Fig. 9)

$$
\begin{aligned}
& \left|m_{E}\right\rangle=\sum_{\alpha, m_{E}^{\prime}}\left[O_{l}^{E}\right]_{\left(\alpha, m_{E}^{\prime}\right), m_{E}}|\alpha\rangle\left|m_{E}^{\prime}\right\rangle, \\
& \left|\bar{m}_{E}\right\rangle=\sum_{\beta, \bar{m}_{E}^{\prime}}\left[\bar{O}_{l}^{E}\right]_{\left(\beta, \bar{m}_{E}^{\prime}\right), \bar{m}_{E}}|\beta\rangle\left|\bar{m}_{E}^{\prime}\right\rangle,
\end{aligned}
$$

where $\left[O_{l}^{E}\right]_{\left(\alpha, m_{E}^{\prime}\right), m_{E}}$ and $\left[\bar{O}_{l}^{E}\right]_{\left(\beta, \bar{m}_{E}^{\prime}\right), \bar{m}_{E}}$ denote the matrix elements of an $s m \times m$ transformation matrices $\left[O_{l}^{E}\right]$ and $\left[\bar{O}_{l}^{E}\right]$ for two different DMRG calculations with distinct system parameters. They are formed by $m$ eigenvectors with the largest eigenvalues of the reduced density matrix of the environment blocks of length $l$. By using Eqs. (A.8) and (A.9), one obtains 


$$
\left\langle\bar{m}_{E} \mid m_{E}\right\rangle=\sum_{\left(\beta, \bar{m}_{E}^{\prime}\right) ;\left(\alpha, m_{E}^{\prime}\right)}\left(\left[\bar{O}_{l}^{E}\right]_{\left(\beta, \bar{m}_{E}^{\prime}\right), \bar{m}_{E}}\right)^{*}\langle\beta \mid \alpha\rangle\left\langle\bar{m}_{E}^{\prime} \mid m_{E}^{\prime}\right\rangle\left[O_{l}^{E}\right]_{\left(\alpha, m_{E}^{\prime}\right), m_{E}}
$$

Following the similar definition in Eq. A.5 , the above expression can be casted into a compact form:

$$
\left[\hat{1}_{l}^{E}\right]=\left[\bar{O}_{l}^{E}\right]^{\dagger}\left(\hat{1} \otimes\left[\hat{1}_{l-1}^{E}\right]\right)\left[O_{l}^{E}\right]
$$

which gives the recursive relations of the $m \times m$ "identity matrices" $\left[\hat{1}_{l}^{E}\right]$ of the environment block of length $l$. For instance, in the cases of $l=2$ and 3 , one has

$$
\begin{gathered}
{\left[\hat{1}_{2}^{E}\right]=\left[\bar{O}_{2}^{E}\right]^{\dagger}(\hat{1} \otimes \hat{1})\left[O_{2}^{E}\right],} \\
{\left[\hat{1}_{3}^{E}\right]=\left[\bar{O}_{3}^{E}\right]^{\dagger}\left(\hat{1} \otimes\left[\hat{1}_{2}^{E}\right]\right)\left[O_{3}^{E}\right] .}
\end{gathered}
$$

Similar recursive relations for the system blocks reads

$$
\left[\hat{1}_{l}^{S}\right]=\left[\bar{O}_{l}^{S}\right]^{\dagger}\left(\left[\hat{1}_{l-1}^{S}\right] \otimes \hat{1}\right)\left[O_{l}^{S}\right]
$$

If we iterate all the way from both ends of the systems by employing the recursive relations in Eqs. A.11 and (A.12), then the matrices $\left[\hat{1}_{\frac{L}{2}-1}^{S}\right]$ and $\left[\hat{1}_{\frac{L}{2}-1}^{E}\right]$ can be constructed eventually. Substituting these results into Eq. (A.6), the value of the ground-state fidelity will be obtained.
[1] M. A. Nielsen and I. L. Chuang, Quantum Computation and Quantum Information (Cambridge University Press, Cambridge, 2000).

[2] S. Sachdev, Quantum Phase Transitions, (Cambridge University Press, Cambridge, 1999).

[3] For a recent review, see L. Amico, R. Fazio, A. Osterloh, and V. Vedral, quant-ph/0703044.

[4] H. T. Quan, Z. Song, X. F. Liu, P. Zanardi, and C. P. Sun, Phys. Rev. Lett. 96, 140604 (2006).

[5] P. Zanardi and N. Paunković, Phys. Rev. E 74, 031123 (2006).

[6] W. L. You, Y. W. Li, and S. J. Gu, Phys. Rev. E 76, 022101 (2007).

[7] P. Zanardi, P. Giorda, and M. Cozzini, Phys. Rev. Lett. 99, 100603 (2007).

[8] H. Q. Zhou, J. H. Zhao, H. L. Wang, and B. Li, arXiv:0711.4651.

[9] P. Zanardi, M. Cozzini, and P. Giorda, J. Stat. Mech. (2007) L02002.

[10] M. Cozzini, P. Giorda, and P. Zanardi, Phys. Rev. B 75, 014439 (2007).

[11] M. Cozzini, R. Ionicioiu, and P. Zanardi, Phys. Rev. B 76, 104420 (2007).

[12] H. Q. Zhou and J. P. Barjaktarevic, arXiv: cond-mat/0701608, H. Q. Zhou, J. H. Zhao, and B. Li, arXiv:0704.2940 H. Q. Zhou, arXiv:0704.2945.

[13] H. Q. Zhou, R. Orús, and G. Vidal, Phys. Rev. Lett. 100, 080601 (2008).

[14] A. Tribedi and I. Bose, Phys. Rev. A 77, 032307 (2008).

[15] P. Buonsante and A. Vezzani, Phys. Rev. Lett. 98, 110601 (2007).

[16] N. Oelkers and J. Links, Phys. Rev. B 75, 115119 (2007).

[17] S. Chen, L. Wang, S. J. Gu, and Y. Wang, Phys. Rev. E 76, 061108 (2007).

[18] W. Q. Ning, S. J. Gu, C. Q. Wu, and H. Q. Lin, arXiv:0708.3178. H. M. Kwok, W. Q. Ning, S. J. Gu, and H. Q. Lin, arXiv:0710.2581.

[19] L. Campos Venuti and P. Zanardi, Phys. Rev. Lett. 99,
095701 (2007).

[20] S. J. Gu, H. M. Kwok, W. Q. Ning, and H. Q. Lin, arXiv:0706.2495

[21] M. F. Yang, Phys. Rev. B 76, 180403(R) (2007), where an error of factor two in some results of the fidelity in the Luttinger liquid phase has been corrected recently [22].

[22] J. O. Fjærestad, arXiv:0712.3439.

[23] Y. C. Tzeng and M. F. Yang, Phys. Rev. A 77, 012311 (2008).

[24] S. Chen, L. Wang, Y. Hao, and Y. Wang, Phys. Rev. A 77, 032111 (2008).

[25] L. Campos Venuti, M. Cozzini, P. Buonsante, F. Massel, N. Bray-Ali, and P. Zanardi, arXiv:0801.2473.

[26] P. Zanardi, H. T. Quan, X. G. Wang, and C. P. Sun, Phys. Rev. A 75, 032109 (2007); P. Zanardi, L. Campos Venuti, and P. Giorda, Phys. Rev. A 76, 062318 (2007); P. Zanardi and M. G. A Paris, arXiv:0708.1089.

[27] N. Paunković and V. R. Vieira, Phys. Rev. E 77, 011129 (2008).

[28] N. Paunković, P. D. Sacramento, P. Nogueira, V. R. Vieira, and V. K. Dugaev, arXiv:0708.3494.

[29] A. Hamma, W. Zhang, S. Haas, and D. A. Lidar, arXiv:0705.0026 [Phys. Rev. B (to be published)].

[30] J. H. Zhao and H. Q. Zhou, arXiv:0803.0814,

[31] S. Yang, S. J. Gu, C. P. Sun, and H. Q. Lin, arXiv:0803.1292

[32] D. F. Abasto, A. Hamma, and P. Zanardi, arXiv:0803.2243

[33] S. R. White, Phys. Rev. Lett. 69, 2863 (1992); Phys. Rev. B 48, 10345 (1993); U. Schollwöck, Rev. Mod. Phys. 77, 259 (2005).

[34] H. J. Schulz, Phys. Rev. B 34, 6372 (1986).

[35] M. den Nijs and K. Rommelse, Phys. Rev. B 40, 4709 (1989).

[36] W. Chen, K. Hida, and B. C. Sanctuary, Phys. Rev. B 67, 104401 (2003).

[37] C. Degli Esposti Boschi, E. Ercolessi, F. Ortolani, and M. Roncaglia, Eur. Phys. J. B 35, 465 (2003). 
[38] L. Campos Venuti, C. Degli Esposti Boschi, M. Roncaglia, and A. Scaramucci, Phys. Rev. A 73, 010303(R) (2006).

[39] L. Campos Venuti, C. Degli Esposti Boschi, E. Ercolessi, G. Morandi, F. Ortolani, S. Pasini, and M. Roncaglia, Eur. Phys. J. B 53, 11 (2006).

[40] S. Lukyanov and A. Zamolodchikov, Nucl. Phys. B 493, 571 (1997).

[41] M. E. Fisher and M. N. Barber, Phys. Rev. Lett. 28, 1516 (1972); M. N. Barber, in Phase Transitions and Critical Phenomena, edited by C. Domb and J. L. Lebowitz (Academic Press, London, 1983), Vol. 8, p. 145.

[42] G. Vidal, J. I. Latorre, E. Rico, and A. Kitaev, Phys. Rev. Lett. 90, 227902 (2003).
[43] C. Holzhey, F. Larsen, and F. Wilczek, Nucl. Phys. B 424, 443 (1994).

[44] V. E. Korepin, Phys. Rev. Lett. 92, 096402 (2004).

[45] P. Calabrese and J. Cardy, J. Stat. Mech. (2004) P06002.

[46] N. Laflorencie, E. S. Sørensen, M. S. Chang, and I. Affleck, Phys. Rev. Lett. 96, 100603 (2006).

[47] H. Q. Zhou, T. Barthel, J. O. Fjærestad, and U. Schollwöck, Phys. Rev. A 74, 050305(R) (2006).

[48] For a recent review, see J. Cardy, arXiv: 0708.2978.

[49] S. Qin, M. Fabrizio, and Lu Yu, Phys. Rev. B 54, 9643 (1996); S. Qin, M. Fabrizio, L. Yu, M. Oshikawa, and I. Affleck, Phys. Rev. B 56, 9766 (1997).

[50] I. P. McCulloch, J. Stat. Mech. (2007) P10014. 